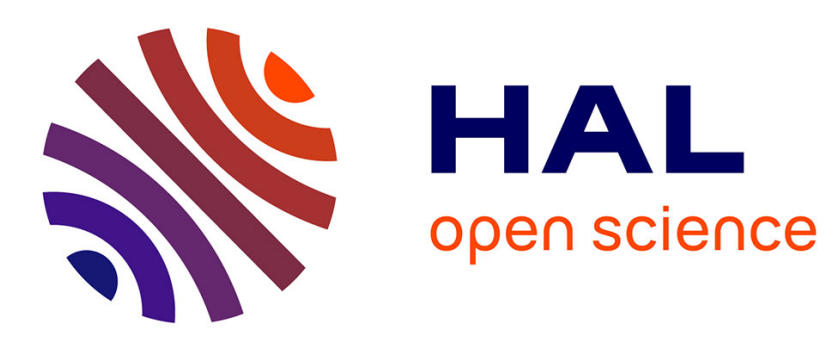

\title{
Politique monétaire optimale et effet de variété en économie ouverte
}

Stéphane Auray, Aurélien Eyquem, Jean-Christophe Poutineau

\section{To cite this version:}

Stéphane Auray, Aurélien Eyquem, Jean-Christophe Poutineau. Politique monétaire optimale et effet de variété en économie ouverte. Economie et Prévision, 2010, 193 (2), pp. 43-58. halshs-00550479

\section{HAL Id: halshs-00550479 \\ https://shs.hal.science/halshs-00550479}

Submitted on 15 Feb 2011

HAL is a multi-disciplinary open access archive for the deposit and dissemination of scientific research documents, whether they are published or not. The documents may come from teaching and research institutions in France or abroad, or from public or private research centers.
L'archive ouverte pluridisciplinaire HAL, est destinée au dépôt et à la diffusion de documents scientifiques de niveau recherche, publiés ou non, émanant des établissements d'enseignement et de recherche français ou étrangers, des laboratoires publics ou privés. 


\title{
Politique monétaire optimale
}

\section{et effet de variété en économie ouverte*}

\author{
Stéphane Auray ${ }^{\dagger} \quad$ Aurélien Eyquem ${ }^{\ddagger}$ \\ Jean-Christophe Poutineau ${ }^{\S}$
}

Septembre 2009

\begin{abstract}
Résumé
Cet article étudie la manière dont la politique monétaire doit être conduite en économie ouverte lorsque l'on prend en compte son influence sur la marge extensive de l'activité. Dans un modèle à deux pays, on montre que, contrairement aux résultats obtenus par Bilbiie, Ghironi et Melitz [2007], la politique monétaire optimale consiste à stabiliser les prix à la consommation, la stabilisation des prix à la production induisant un ajustement insuffisant du taux de change réel. D'autre part, lorsque l'on tient compte de la marge intensive de l'activité, l'adoption d'un régime de change fixe entraîne une sur-volatilité macroéconomique qui génère des coûts en termes de bien-être lorsque les relations commerciales sont importantes et que les heures travaillées sont fortement élastiques au salaire réel.
\end{abstract}

Mots clés : politique monétaire optimale, économie ouverte, effet de variété, marge extensive. Classification JEL : E51, E58, F36, F41.

\footnotetext{
*Nous tenons à remercier Pierre Malgrange ainsi que deux rapporteurs anonymes pour leurs précieux conseils et suggestions qui ont grandement contribué à l'amélioration de ce texte.

${ }^{\dagger}$ EQUIPPE, EA4018, Universités Lille Nord de France (ULCO), GREDI, Université de Sherbrooke et CIRPÉE, Canada. Email : stephane auray@gmail.com.

${ }^{\ddagger}$ GATE, UMR 5824, Université de Lyon, Ecole Normale Supérieure LSH, France et GREDI, Université de Sherbrooke, Canada.

${ }^{\S}$ CREM, UMR 6211, Université de Rennes 1 et Ecole Normale Supérieure de Cachan, France.
} 


\section{Introduction}

La politique monétaire agit sur l'activité économique à travers deux marges : la marge intensive - c'est-à-dire la quantité des biens et services produits dans l'économie - et la marge extensive - c'est-à-dire la création de nouvelles activités (entreprises ou nouveaux produits). D'un point de vue empirique, Bergin et Corsetti [2005] montrent, à l'aide d'un modèle SVAR, que cette seconde marge joue un rôle significatif dans la politique monétaire américaine. Sur le plan analytique Bilbiie et al. [2007] montrent que la politique monétaire est susceptible d'agir sur le nombre de firmes présentes sur un marché du fait de son influence sur la rentabilité des investissements dans la création de nouvelles firmes. Dans ce cadre, la dynamique du taux d'intérêt réel est cruciale pour l'arbitrage intertemporel auquel font face les firmes lorsqu'elles décident d'investir dans la création de nouvelles variétés de biens. Bilbiie et al. [2007] montrent en particulier que la politique monétaire optimale en économie fermée consiste à stabiliser l'inflation des prix à la production.

Cet article étend l'analyse de la politique monétaire optimale au cadre d'une économie ouverte. Dans cet environnement autorisant les échanges commerciaux et financiers, plusieurs questions nouvelles se posent. L'existence de liens commerciaux conduit-il, comme dans le cas standard, à stabiliser l'inflation des prix à la production? Dans la mesure où les rendements financiers affectent de manière importante les décisions en matière de création de firmes, l'intégration financière internationale conduit-elle à de nouvelles prescriptions en matière de politique monétaire optimale? Enfin, les pertes de bien-être traditionnellement attachées à l'abandon du taux de change nominal comme variable d'ajustement externe sont-elles réduites ou amplifiées par la prise en compte de la marge extensive? Le modèle développé pour répondre à ces questions s'appuie sur les travaux de Bilbiie et al. [2007] : les prix sont rigides de par l'existence de coûts d'ajustement à la Rotemberg [1984] et le nombre de variétés disponibles dans chaque économie varie de manière endogène. Ce cadre est étoffé pour aborder les aspects liés à l'ouverture des économies. Le monde se compose de deux pays, les marchés financiers sont parfaitement intégrés et toutes les variétés de biens sont échangées moyennant le paiement de coûts de transport, ce qui fait apparaître un biais en faveur des variétés produites localement. La politique monétaire optimale est déterminée dans deux situations polaires : $(i)$ en régime de change flexible et (ii) en régime de change fixe. 
Cet article montre que la politique monétaire optimale ne consiste pas à stabiliser intégralement l'inflation des prix à la production, contrairement au résultat de Bilbiie et al. [2007] en économie fermée. Ce résultat provient de l'externalité générée par les politiques monétaires sur les termes de l'échange. En effet, une stabilisation parfaite des taux d'inflation des prix à la production conduit à une insuffisante variabilité des termes de l'échange, pourtant nécessaire à l'ajustement externe des économies. Ainsi, l'objectif de stabilité des prix à la production n'est plus l'objectif optimal de la politique monétaire en économie ouverte.

Un second résultat, relatif aux coûts/bénéfices liés à l'adoption d'un régime de change fixe, émerge de l'analyse. Bergin et Lin [2008] montrent que les régimes de change fixe et plus encore les unions monétaires, permettent une réduction de l'incertitude dans le mécanisme de fixation des prix. Ceci conduit alors à une augmentation sensible du niveau des échanges commerciaux par le biais de la marge extensive. Dans cet article, nous ne considérons pas les effets de premier ordre mais nous nous intéressons aux effets des régimes de change sur la volatilité macroéconomique. Les régimes de change fixe conduisent à une survolatilité macroéconomique, liée à la restriction des instruments de politique monétaire, et ce même lorsque la politique monétaire commune est fixée de manière coopérative. Ainsi, les coûts d'adoption d'un régime de change fixe représentent entre $0.01 \%$ et $0.1 \%$ de consommation permanente selon les valeurs des paramètres. Ces résultats relatifs à la supériorité des régimes de change flexible rejoignent qualitativement et quantitativement ceux de Devereux et Engel [2003] et de Gali et Monacelli [2005]. Enfin, la prise en considération de la marge extensive est susceptible de réduire ou de magnifier les coûts d'adoption d'un système de change fixe par rapport à l'analyse standard.

L'article s'organise de la manière suivante. La section 2 détaille le cadre d'analyse. La section 3 présente la dynamique des économies en régime de change flexible et fixe lorsque la politique monétaire est déterminée de manière optimale. La section 4 présente une analyse des différentes situations envisagées en termes de bien-être. La section 5 conclut.

\section{Un monde à deux pays}

Ce premier paragraphe présente les comportements des ménages et des entreprises ainsi que les conditions d'équilibre général dans un monde à deux pays. Chaque pays représente la moitié du monde et est peuplé d'une masse unitaire de consommateurs. Dans l'économie domestique, $n_{t}$ 
firmes opèrent sur le marché des biens et services en situation de concurrence monopolistique à la date $t$ en utilisant des facteurs de production nationaux. De manière symétrique, dans l'économie étrangère, $n_{t}^{*}$ firmes opèrent sur le marché des biens et services à la date $t$. Les ménages ont accès à trois actifs financiers : les parts d'un fonds mutuel d'investissement national et des bons du trésor d'une période émis dans chaque pays.

\subsection{Les ménages}

Le ménage représentatif $j \in[0,1]$ du pays domestique maximise son bien-être,

$$
\Omega_{t}(j)=E_{0}\left\{\sum_{t=0}^{\infty} \beta^{t} u\left(c_{t}(j), \ell_{t}(j)\right)\right\},
$$

sous la contrainte,

$$
\begin{aligned}
& b_{t+1}(j)+e_{t} b_{t+1}^{*}(j)+p_{c, t}\left(v_{t}\left(n_{t}+n_{e, t}\right) x_{t+1}(j)+c_{t}(j)\right)= \\
& \quad\left(1+i_{t-1}\right) b_{t}(j)+e_{t}\left(1+i_{t-1}^{*}\right) b_{t}^{*}(j)+p_{c, t}\left(d_{t}+v_{t}\right) n_{t} x_{t}(j)+\left(1-\zeta_{t}\right) w_{t} \ell_{t}(j)+\gamma_{t}(j) .
\end{aligned}
$$

Dans ces expressions, $\beta$ représente le facteur d'escompte, $c_{t}(j)$ le panier de consommation choisi par le ménage $j$ et $\ell_{t}(j)$ son offre de travail. La variable $e_{t}$ est le taux de change nominal coté à l'incertain pour l'économie domestique, $p_{c, t}$ représente l'indice des prix domestiques à la consommation et $w_{t}$ le salaire horaire nominal. De plus, $\zeta_{t}$ est une taxe ayant pour objet de corriger les distorsions liées à la structure concurrentielle imparfaite du marché des biens et services et $\gamma_{t}(j)$ est un transfert forfaitaire. Le ménage $j$ détient trois types d'actifs : les parts d'un fonds mutuel d'investissement domestique en quantité $x_{t+1}(j)$, des bons nationaux d'une période en quantité $b_{t+1}(j)$, offrant une rémunération $i_{t-1}$ entre les périodes $t-1$ et $t$ et des bons étrangers d'une période en quantité $b_{t+1}^{*}(j)$, offrant une rémunération $i_{t-1}^{*}$ entre les périodes $t-1$ et $t$. La valeur du fonds à la date $t$ dépend de la valeur réelle moyenne des firmes $v_{t}$ et du nombre de firmes sur le marché à la date $t$, notée $n_{t}+n_{e, t}$, où $n_{e, t}$ représente les firmes entrées sur le marché à cette date. A la période $t$, le ménage représentatif détermine donc la fraction optimale $x_{t+1}(j)$ qu'il souhaite détenir du fonds national compte tenu de la valeur du fonds à la date $t v_{t}\left(n_{t}+n_{e, t}\right)$ et du montant réel moyen de dividendes à cette date, $d_{t}$. Les ménages de l'économie étrangère se comportent de manière symétrique. L'analyse se concentre 
donc sur le comportement des ménages domestiques.

Les conditions du premier ordre pour le ménage représentatif de l'économie domestique par rapport à $c_{t}(j), \ell_{t}(j), b_{t+1}(j), b_{t+1}^{*}(j)$ et $x_{t+1}(j)$ impliquent,

$$
\begin{aligned}
-\frac{u_{\ell, t}}{u_{c, t}}-\left(1-\zeta_{t}\right) \varpi_{t} & =0, \\
\beta E_{t}\left\{\frac{u_{c, t+1}}{u_{c, t}}\left(1+r_{t}\right)\right\} & =1, \\
\left(1+i_{t}\right)-\left(1+i_{t}^{*}\right) \frac{E_{t}\left\{e_{t+1}\right\}}{e_{t}} & =0, \\
v_{t}-\beta(1-\delta) E_{t}\left\{\frac{u_{c, t+1}}{u_{c, t}}\left(d_{t+1}+v_{t+1}\right)\right\} & =0,
\end{aligned}
$$

où $\varpi_{t}=\frac{w_{t}}{p_{c, t}}$ est le salaire réel, $1+r_{t}=\frac{1+i_{t}}{1+\pi_{c, t+1}}$ le taux d'intérêt réel, $\pi_{c, t}=\frac{p_{c, t}}{p_{c, t-1}}-1$ le taux d'inflation des prix à la consommation et où l'équation décrivant la dynamique des firmes dans l'économie a été utilisée pour obtenir l'équation (4) ${ }^{1}$. L'équation (1) décrit l'arbitrage travail/loisir des ménages et l'équation (2) est une équation d'Euler standard décrivant l'évolution de la consommation agrégée. L'équation (3) indique que la parité des taux d'intérêt est vérifée et l'équation (4), combinée à l'équation (2),

$$
v_{t}-(1-\delta) E_{t}\left\{\frac{d_{t+1}+v_{t+1}}{1+r_{t}}\right\}=0
$$

indique que les rendements réels associés à la détention de parts du fonds mutuel doivent égaliser à l'équilibre les rendements réels associés à la détention des autres actifs disponibles dans l'économie, en particulier les bons du trésor nationaux ${ }^{2}$. Une condition identique à l'équation (2) est vérifiée pour les ménages étrangers. Il est ainsi possible de montrer que les marchés financiers sont complets au niveau international en combinant les deux relations d'Euler et la relation de parité des taux d'intérêt, ce qui donne,

$$
\frac{u_{c, t}^{*}}{u_{c, t}}=\frac{e_{t} p_{c, t}^{*}}{p_{c, t}}=q_{t}
$$

où $q_{t}$ est le taux de change réel coté à l'incertain pour l'économie domestique.

1. $\delta$ représente la proportion de firmes disparaissant à chaque période

2. Il est à noter que combiner cette équation avec l'équation (3) permet également d'obtenir une relation d'arbitrage entre les rendements des parts du fonds et les bons du trésor étrangers. 
Dans chaque pays, à la date $t$, les consommateurs de l'économie domestique ont accès à une quantité $n_{t}$ de variétés (indexées en $\omega$ ) de biens et services produits dans l'économie domestique et à $n_{t}^{*}$ types de variétés produites dans l'économie étrangère. Le panier de biens consommé dans l'économie domestique est une combinaison de ces $\left(n_{t}+n_{t}^{*}\right)$ variétés avec une élasticité de substitution entre les variétés $\sigma>1$. La consommation totale du ménage $j$ s'écrit,

$$
c_{t}(j)=\left[\int_{0}^{n_{t}} c_{d, t}(\omega, j)^{\frac{\sigma-1}{\sigma}} d \omega+\int_{0}^{n_{t}^{*}} c_{m, t}\left(\omega^{*}, j\right)^{\frac{\sigma-1}{\sigma}} d \omega^{*}\right]^{\frac{\sigma}{\sigma-1}}
$$

et l'indice de prix correspondant,

$$
p_{c, t}=\left[\int_{0}^{n_{t}} p_{t}(\omega)^{1-\sigma} d \omega+\int_{0}^{n_{t}^{*}} p_{x, t}^{*}\left(\omega^{*}\right)^{1-\sigma} d \omega^{*}\right]^{\frac{1}{1-\sigma}} .
$$

Les expressions sont comparables pour l'économie étrangère. On suppose que le prix des biens importés $p_{x, t}^{*}\left(\omega^{*}\right)$ (respectivement $p_{x, t}(\omega)$ ) et consommés dans l'économie domestique (resp. étrangère) sont affectés par des coûts de transport iceberg : les consommateurs domestiques doivent acheter une quantité $(1+\tau)$ de chaque type de bien étranger pour en consommer une unité. En supposant que les producteurs se fondent sur le prix de vente domestique $p_{t}^{*}\left(\omega^{*}\right)$ pour déterminer leur prix de vente à l'exportation $p_{x, t}^{*}\left(\omega^{*}\right)$, on obtient,

$$
p_{x, t}^{*}\left(\omega^{*}\right)=(1+\tau) e_{t} p_{t}^{*}\left(\omega^{*}\right) .
$$

Les demandes optimales de variétés émanant des ménages domestiques sont donc,

$$
\begin{gathered}
c_{d, t}(\omega, j)=\rho_{t}(\omega)^{-\sigma} c_{t}(j), \\
c_{m, t}\left(\omega^{*}, j\right)=\left((1+\tau) q_{t} \rho_{t}^{*}\left(\omega^{*}\right)\right)^{-\sigma} c_{t}(j),
\end{gathered}
$$

où $\rho_{t}(\omega)=\frac{p_{t}(\omega)}{p_{c, t}}$ et $\rho_{t}^{*}\left(\omega^{*}\right)=\frac{p_{t}^{*}\left(\omega^{*}\right)}{p_{c, t}^{*}}$ sont les prix réels des biens $\omega$ et $\omega^{*}$. 


\section{$2.2 \quad$ Les firmes}

A chaque période $t$, il existe deux types de firmes dans l'économie domestique ${ }^{3}: n_{t}$ firmes déjà présentes dans l'économie au début de la période et $n_{e, t}$ firmes qui sont créées en cours de période. A la fin de chaque période, une proportion $\delta \in[0,1]$ de firmes existantes disparait. On suppose que l'entrée d'une firme sur le marché s'effectue une période avant le début de la production de ses biens, la création de l'entreprise étant consommatrice de temps lors de la première période. De ce fait le nombre de firmes produisant des biens à la période $t$ est donné par,

$$
n_{t}=(1-\delta)\left(n_{t-1}+n_{e, t-1}\right)
$$

Chaque firme est spécialisée dans la production d'un bien imparfaitement substituable aux autres biens disponibles à la consommation dans l'économie. La fonction de production de la firme représentative domestique $\omega$ est,

$$
y_{t}(\omega)=z_{t} \ell_{t}^{d}(\omega)
$$

où $z_{t}$ est un choc de productivité commun à toutes les firmes domestiques tel que,

$$
\log z\left({ }_{t+1}\right)=\rho_{z} \log \left(z_{t}\right)+\xi_{z, t},
$$

et $\ell_{t}^{d}(\omega)$ est la demande de travail de la firme représentative $\omega$.

Les ajustements de prix par les entreprises engendrent le paiement de coûts à la Rotemberg [1984], payés sous forme de biens de consommation finale. Pour une firme $\omega$, le coût d'ajustement est donc,

$$
\Gamma_{t}(\omega)=\frac{\kappa}{2}\left(\frac{p_{t}(\omega)}{p_{t-1}(\omega)}-1\right)^{2} \rho_{t}(\omega) y_{t}^{d}(\omega)
$$

De ce fait, la demande s'adressant à la firme $\omega$ s'écrit,

$$
y_{t}^{d}(\omega)=\rho_{t}(\omega)^{-\sigma}\left[\left(c_{t}+\Gamma_{t}\right)+\left((1+\tau) q_{t}^{-1}\right)^{-\sigma}\left(c_{t}^{*}+\Gamma_{t}^{*}\right)\right] \text {, }
$$

avec $c_{t}=\int_{0}^{1} c_{t}(j) d j, c_{t}^{*}=\int_{0}^{1} c_{t}^{*}(j) d j, \Gamma_{t}=\int_{0}^{n_{t}} \Gamma_{t}(\omega) d \omega$ et $\Gamma_{t}^{*}=\int_{0}^{n_{t}^{*}} \Gamma_{t}^{*}\left(\omega^{*}\right) d \omega^{*}$.

3. L'analyse se concentre sur l'économie domestique sachant que des relations similaires définissent le comportement des firmes étrangères. 
A la période $t$, la firme représentative $\omega$ choisit le prix de vente $p_{t}(\omega)$ lui permettant de maximiser $d_{t}(\omega)+v_{t}(\omega)$, avec,

$$
\begin{gathered}
v_{t}(\omega)=E_{t}\left\{\sum_{s=1}^{\infty}(\beta(1-\delta))^{s}\left(\frac{u_{c, t+s}}{u_{c, t}} d_{t+s}(\omega)\right)\right\} \\
d_{t}(\omega)=\rho_{t}(\omega) y_{t}^{d}(\omega)-\frac{\kappa}{2}\left(\frac{p_{t}(\omega)}{p_{t-1}(\omega)}-1\right)^{2} \rho_{t}(\omega) y_{t}^{d}(\omega)-\frac{\varpi_{t}}{z_{t}} y_{t}^{d}(\omega) .
\end{gathered}
$$

La tarification optimale qui en résulte est la suivante,

$$
\rho_{t}(\omega)=\frac{\sigma}{(\sigma-1)\left(1-\frac{\kappa}{2} \pi_{t}^{2}\right)+\kappa \eta_{t}} \frac{\varpi_{t}}{z_{t}}
$$

avec,

$$
\eta_{t}=\left(1+\pi_{t}\right) \pi_{t}-\beta(1-\delta) E_{t}\left\{\frac{\pi_{t+1}^{2}\left(1+\pi_{t+1}\right)^{2} y_{t+1} u_{c, t+1}}{\left(1+\pi_{c, t+1}\right) y_{t} u_{c, t}}\right\}
$$

A la période $t, n_{e, t}$ nouvelles firmes entrent sur le marché tant que leur valeur $v_{t}$ est supérieure au coût de création de la firme, calculé en termes de travail efficace $f_{e, t} \frac{\varpi_{t}}{z_{t}}$. La variable $f_{e, t}$ peut s'interpréter comme un paramètre de politique structurelle ou comme un choc exogène que subit l'économie (voir Bilbiie et al. [2007]) ${ }^{4}$. L'entrée de nouvelles firmes sur le marché s'arrête donc dès que,

$$
v_{t}=f_{e, t} \frac{\varpi_{t}}{z_{t}}
$$

\subsection{Agrégation et Equilibre}

Le modèle est résolu en supposant que les firmes adoptent un comportement symétrique, c'est-à-dire,

$$
\rho_{t}(\omega)=\rho_{t}, \quad \rho_{t}^{*}\left(\omega^{*}\right)=\rho_{t}^{*}
$$

Par conséquent, le lien entre le taux d'inflation des prix à la production et le taux d'inflation des prix à la consommation est,

$$
\frac{1+\pi_{t}}{1+\pi_{c, t}}=\frac{\rho_{t}}{\rho_{t-1}}, \quad \frac{1+\pi_{t}^{*}}{1+\pi_{c, t}^{*}}=\frac{\rho_{t}^{*}}{\rho_{t-1}^{*}} .
$$

4. Dans cette économie, un choc négatif sur $f_{e, t}$ produit des effets strictement symétriques à ceux d'un choc positif de productivité. L'étude spécifique de ce choc ne présente donc qu'un intérêt limité dans le cadre de cet article. 
De plus, compte tenu de la forme des indices de prix à la consommation, l'effet de variété est déterminé par,

$$
n_{t} \rho_{t}^{1-\sigma}+\phi q_{t}^{1-\sigma} n_{t}^{*} \rho_{t}^{* 1-\sigma}=1, \quad n_{t}^{*} \rho_{t}^{* 1-\sigma}+\phi q_{t}^{\sigma-1} n_{t} \rho_{t}^{1-\sigma}=1
$$

où $\phi=(1+\tau)^{1-\sigma}$ détermine la part relative des biens nationaux et importés dans la consommation du ménage représentatif.

Enfin, la mesure du produit national est la suivante,

$$
y_{t}=\int_{0}^{n_{t}} \rho_{t}(\omega) y_{t}(\omega) d \omega, \quad y_{t}^{*}=\int_{0}^{n_{t}^{*}} \rho_{t}^{*}\left(\omega^{*}\right) y_{t}^{*}\left(\omega^{*}\right) d \omega^{*}
$$

Etant données ces relations, l'équilibre de l'économie peut être défini comme une séquence de quantités,

$$
\left\{\mathcal{Q}_{t}\right\}_{t=0}^{\infty}=\left\{y_{t}, y_{t}^{*}, c_{t}, c_{t}^{*}, \ell_{t}, \ell_{t}^{*}, \ell_{t}^{d}, \ell_{t}^{d *}, n_{t}, n_{t}^{*}, n_{e, t}, n_{e, t}^{*}\right\}_{t=0}^{\infty},
$$

et une séquence de prix,

$$
\left\{\mathcal{P}_{t}\right\}_{t=0}^{\infty}=\left\{\rho_{t}, \rho_{t}^{*}, \eta_{t}, \eta_{t}^{*}, \varpi_{t}, \varpi_{t}^{*}, v_{t}, v_{t}^{*}, d_{t}, d_{t}^{*}, \pi_{t}, \pi_{t}^{*}, \pi_{c, t}^{*}, \pi_{c, t}, q_{t}\right\}_{t=0}^{\infty},
$$

tels que :

(i) pour une séquence de prix donnée $\left\{\mathcal{P}_{t}\right\}_{t=0}^{\infty}$ et la réalisation des chocs $\left\{\mathcal{S}_{t}\right\}_{t=0}^{\infty}=\left\{z_{t}, z_{t}^{*}\right\}_{t=0}^{\infty}$, la séquence $\left\{\mathcal{Q}_{t}\right\}_{t=0}^{\infty}$ respecte les conditions du premier ordre des ménages domestiques et étrangers et maximise le profit des firmes domestiques et étrangères.

(ii) pour une séquence de quantités donnée $\left\{\mathcal{Q}_{t}\right\}_{t=0}^{\infty}$ et la réalisation des chocs $\left\{\mathcal{S}_{t}\right\}_{t=0}^{\infty}=$ $\left\{z_{t}, z_{t}^{*}\right\}_{t=0}^{\infty}$, la sequence $\left\{\mathcal{P}_{t}\right\}_{t=0}^{\infty}$ garantit :

- l'équilibre du marché du travail,

$$
\begin{gathered}
\ell_{t}=\int_{0}^{1} \ell_{t}(j) d j=\int_{0}^{n_{t}} \ell_{t}^{d}(\omega) d \omega+z_{t}^{-1} f_{e, t} n_{e, t}, \\
\ell_{t}^{*}=\int_{0}^{1} \ell_{t}^{*}\left(j^{*}\right) d j^{*}=\int_{0}^{n_{t}^{*}} \ell_{t}^{* d}\left(\omega^{*}\right) d \omega^{*}+z_{t}^{*^{-1}} f_{e, t} n_{e, t}^{*},
\end{gathered}
$$


- l'équilibre du marché des biens et services,

$$
\begin{aligned}
y_{t} & =n_{t} \rho_{t}^{1-\sigma}\left(c_{t}+\frac{\kappa}{2} \pi_{t}^{2} y_{t}+\phi q_{t}^{\sigma-1}\left(c_{t}^{*}+\frac{\kappa}{2} \pi_{t}^{* 2} y_{t}^{*}\right)\right), \\
y_{t}^{*} & =n_{t}^{*} \rho_{t}^{* 1-\sigma}\left(c_{t}^{*}+\frac{\kappa}{2} \pi_{t}^{* 2} y_{t}^{*}+\phi q_{t}^{1-\sigma}\left(c_{t}+\frac{\kappa}{2} \pi_{t}^{2} y_{t}\right)\right) .
\end{aligned}
$$

En utilisant ces relations d'équilibre, il est alors possible de simplifier les relations agrégées dans les deux économies. Les fonctions de production agrégées s'écrivent,

$$
y_{t}=n_{t} \rho_{t} z_{t} \ell_{t}^{d}, \quad y_{t}^{*}=n_{t}^{*} \rho_{t}^{*} z_{t}^{*} \ell_{t}^{d *}
$$

L'équilibre du marché du travail devient,

$$
z_{t} \ell_{t}=\frac{y_{t}}{\rho_{t}}+f_{e, t} n_{e, t}, \quad z_{t}^{*} \ell_{t}^{*}=\frac{y_{t}^{*}}{\rho_{t}^{*}}+f_{e, t}^{*} n_{e, t}^{*}
$$

et les dividendes évoluent selon,

$$
\begin{aligned}
d_{t} & =\frac{y_{t}}{n_{t}}\left(1-\frac{\kappa}{2} \pi_{t}^{2}-\frac{(\sigma-1)\left(1-\frac{\kappa}{2} \pi_{t}^{2}\right)+\kappa \eta_{t}}{\sigma}\right), \\
d_{t}^{*} & =\frac{y_{t}^{*}}{n_{t}^{*}}\left(1-\frac{\kappa}{2} \pi_{t}^{* 2}-\frac{(\sigma-1)\left(1-\frac{\kappa}{2} \pi_{t}^{* 2}\right)+\kappa \eta_{t}^{*}}{\sigma}\right) .
\end{aligned}
$$

\section{Politique monétaire optimale}

Cette section présente le problème de politique monétaire optimale dans le monde à deux pays décrit dans la section précédente. Avant de présenter en détails le problème de Ramsey, il convient de préciser que la politique monétaire n'a pas d'effet sur l'état stationnaire des économies, que nous décrivons dans le paragraphe suivant.

\subsection{L'état stationnaire}

L'analyse est conduite autour d'un état stationnaire symétrique et Pareto-efficace. La symétrie de l'état stationnaire impose $q=1$. De plus, on pose $z=1$. Par ailleurs, on considère une situation où les coûts d'entrée sont constants, soit $f_{e, t}=f_{e}$. Enfin, par définition, à l'état stationnaire, on a $\pi=\pi^{*}=0$, ce qui implique $\rho=\frac{\sigma}{\sigma-1} \varpi$. Afin de rétablir une allocation Paretienne des res- 
sources à l'état stationnaire, la valeur de la taxe sur le travail est donc $\zeta=(1-\sigma)^{-1}$.

En posant la forme fonctionnelle suivante pour la fonction d'utilité du ménage représentatif,

$$
u\left(c_{t}(j), \ell_{t}(j)\right)=\frac{c_{t}(j)^{1-\gamma}}{1-\gamma}-\frac{\ell_{t}(j)^{1+\psi}}{1+\psi}
$$

les relations d'équilibre à l'état stationnaire donnent,

$$
\begin{gathered}
\varpi=\frac{\sigma-1}{\sigma}, n=\frac{1}{1+\phi}, n_{e}=\frac{\delta}{(1-\delta)(1+\phi)}, c=(1+\varphi)^{-\frac{\psi}{\gamma+\psi}}, \ell=(1+\varphi)^{\frac{\gamma}{\gamma+\psi}}, \\
y=c, d=\frac{c}{\sigma}, v=\frac{\beta(1-\delta)}{(1-\beta(1-\delta))} \frac{c}{\sigma}, f_{e}=\frac{\beta(1-\delta)}{(1-\beta(1-\delta))(\sigma-1)} c,
\end{gathered}
$$

où $\varphi=\frac{\beta \delta}{(\sigma-1)(1+\phi)(1-\beta(1-\delta))}$.

\subsection{Principe et démarche}

Nous nous intéressons maintenant à la dynamique des économies autour de cet état stationnaire lorsque la politique monétaire est déterminée de manière optimale. Il s'agit de déterminer la politique monétaire avec engagement préalable qui maximise le bien-être agrégé au niveau international, c'est-à-dire une politique monétaire coopérative. La fonction d'utilité des ménages constituant un objectif naturel de la politique économique conduite par les autorités, la solution du problème de Ramsey est donc une séquence,

$$
\{\Xi\}_{t=0}^{\infty}=\left\{\epsilon_{t}, \epsilon_{t}^{*}, i_{t}, i_{t}^{*}\right\}_{t=0}^{\infty}
$$

où,

$$
\begin{gathered}
\left\{\epsilon_{t}\right\}_{t=0}^{\infty}=\left\{c_{t}, \ell_{t}, \varpi_{t}, y_{t}, n_{t}, n_{e, t}, d_{t}, v_{t}, \rho_{t}, \eta_{t}, \pi_{t}, \pi_{c, t}\right\}_{t=0}^{\infty}, \\
\left\{\epsilon_{t}^{*}\right\}_{t=0}^{\infty}=\left\{c_{t}^{*}, \ell_{t}^{*}, \varpi_{t}^{*}, y_{t}^{*}, n_{t}^{*}, n_{e, t}^{*}, d_{t}^{*}, v_{t}^{*}, \rho_{t}^{*}, \eta_{t}^{*}, \pi_{t}^{*}, \pi_{c, t}^{*}\right\}_{t=0}^{\infty},
\end{gathered}
$$

qui résout le problème suivant,

$$
\underset{\Xi_{t}}{\operatorname{Max}} \Phi_{t}=E_{0} \sum_{t=0}^{\infty} \beta^{t}\left\{\int_{0}^{1} u\left(c_{t}(j), \ell_{t}(j)\right) d j+\int_{0}^{1} u\left(c_{t}^{*}(j), \ell_{t}^{*}(j)\right) d j\right\}
$$


sous contrainte des équations (5)-(17), présentées en annexe et décrivant la dynamique des économies.

Ce problème de politique monétaire avec engagement préalable fait apparaître une incohérence temporelle, dans la mesure où les autorités peuvent choisir la valeur de leurs instruments de politique monétaire à la période zéro après que les agents aient formé leurs anticipations d'inflation. Par conséquent, après la première période, les autorités sont amenées à changer leurs politiques économiques car les agents révisent leurs anticipations sur la base de l'évolution passée de l'économie, qui révèle la fonction objectif des autorités. La différence de comportement entre la première période et l'ensemble des autres périodes peut être ignorée en supposant que les autorités choisissent leur politique économique conformément à un engagement préalable similaire à celui qu'elles prendront dans le futur. S'obligeant à honorer cet engagement préalable, la manipulation des anticipations d'inflation devient impossible et la cohérence temporelle de la politique économique conduite est restaurée. Cette manière de résoudre le problème de Ramsey est conforme à la perspective dite atemporelle présentée par Woodford (2003) et garantit l'unicité de la politique monétaire optimale adoptée dans le temps.

\subsection{La dynamique des économies}

La politique monétaire optimale est donc dérivée en adoptant une perspective atemporelle. Elle est calculée en résolvant le problème de Ramsey en niveau ${ }^{5}$ et en réalisant une approximation à l'ordre deux des conditions d'équilibre ainsi obtenues ${ }^{6}$ autour de l'état stationnaire efficace.

On résout alternativement le problème de Ramsey en régime de change flexible et en régime de change fixe. On retient une spécification trimestrielle du modèle. Les valeurs numériques retenues pour les paramètres sont les suivantes : le facteur d'actualisation est $\beta=0.99$, ce qui implique un taux d'intérêt réel annuel de $4 \%$ environ à l'état stationnaire. La proportion de firmes disparaissant au terme de chaque période est $\delta=0.025$. Le paramètre de persistance d'inflation apparaissant dans la règle d'ajustement du prix des biens est $\kappa=77$ (voir Bilbiie et al. [2007]). L'élasticité de substitution entre les variétés détermine le taux de marge à l'état stationnaire (compensé par la taxe optimale sur les salaires). En suivant Bilbiie et al. [2007], on suppose $\sigma=4$, ce qui implique un taux de marge de $33 \%$ à l'état stationnaire. Le coût de

5. On utilise pour cela la symbolic toolbox de Matlab.

6. On utilise pour cela l'algorithme basé sur la méthode de Schmitt-Grohe et Uribe [2004]. 
transport iceberg est $\tau=0.5$, ce qui correspond à la valeur moyenne proposée par Corsetti, Martin et Pesenti [2007]. Cette valeur implique que les importations représentent environ 22.9\% de la consommation des ménages ${ }^{7}$. Le paramètre d'aversion au risque est fixé à $\gamma=2$. L'inverse de l'élasticité frichienne est $\psi^{-1}=0.5$, ce qui entre dans l'intervalle proposé par Canzoneri, Cumby et Diba [2007]. Finalement, la persistance des chocs sur la productivité du travail est $\rho_{z}=0.9$ et l'écart-type des innovations est $\sigma\left(\xi_{z, t}\right)=0.7 \%$. On considère une version symétrique du modèle. Les paramètres sont donc identiques pour l'économie étrangère.

Les graphiques 1 et 2 décrivent la réponse des deux économies en régime de change flexible à la suite d'un choc de productivité positif et asymétrique d'1\% se produisant dans l'économie domestique $^{8}$. A des fins de comparaison, les graphiques reportent également la dynamique des économies en régime de change flexible lorsque les prix sont flexibles.

A la suite d'un choc de productivité domestique, la production s'accroît sous l'impact des gains de productivité agissant à la fois à travers la marge intensive (la production de variétés déjà disponibles dans l'économie) et à travers la marge extensive, c'est-à-dire la création de nouvelles firmes. Pour une valeur des firmes données, l'augmentation de la productivité accroît l'efficacité des heures travaillées employées à créer de nouvelles firmes, ce qui, par le biais de la condition de libre entrée, accroît le nombre de firmes opérant dans l'économie domestique. Comme dans un modèle avec du capital physique, les heures travaillées augmentent pour répondre aux besoins des firmes existantes et à ceux des firmes qui entrent sur le marché. La consommation augmente dans les deux économies, bien que plus sensiblement dans l'économie domestique sous l'effet des gains de productivité. La transmission du choc à l'économie étrangère passe par le taux de change réel, qui augmente. Cette amélioration de la compétitivité des firmes domestiques engendre un effet de substitution des consommations et provoque un déficit de la balance commerciale dans l'économie étrangère. La demande totale de variétés étrangères baisse, ce qui entraîne une baisse de la production étrangère. L'augmentation du prix relatif des variétés étrangères les rend moins compétitives et décourage l'investissement dans la création de nouvelles variétés.

En l'absence de politique monétaire active, l'existence de coûts d'ajustements génère une déflation dans l'économie domestique, qui correspond à un décalage dans le temps entre la matérialisation des gains de productivité dont bénéficient les firmes et leurs répercussions sur les

7. La part des importations est donnée par $\frac{\phi}{1+\phi} \simeq 0.229$, avec $\phi=(1+\tau)^{1-\sigma} \simeq 0.296$.

8. L'analyse d'un choc de productivité se produisant dans l'économie étrangère est rigoureusement symétrique. 
FIgURE 1 - Réponse à un choc domestique de productivité (1) - taux de change flexible.
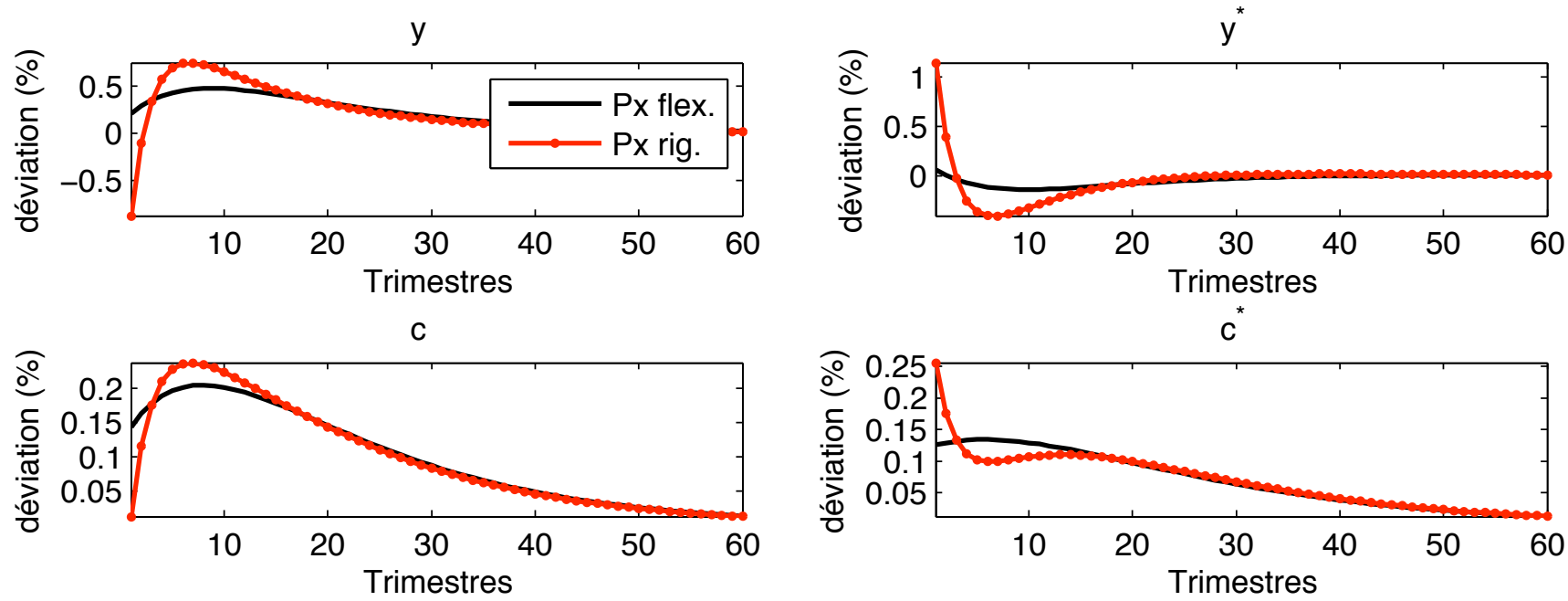

$\mathrm{L}$
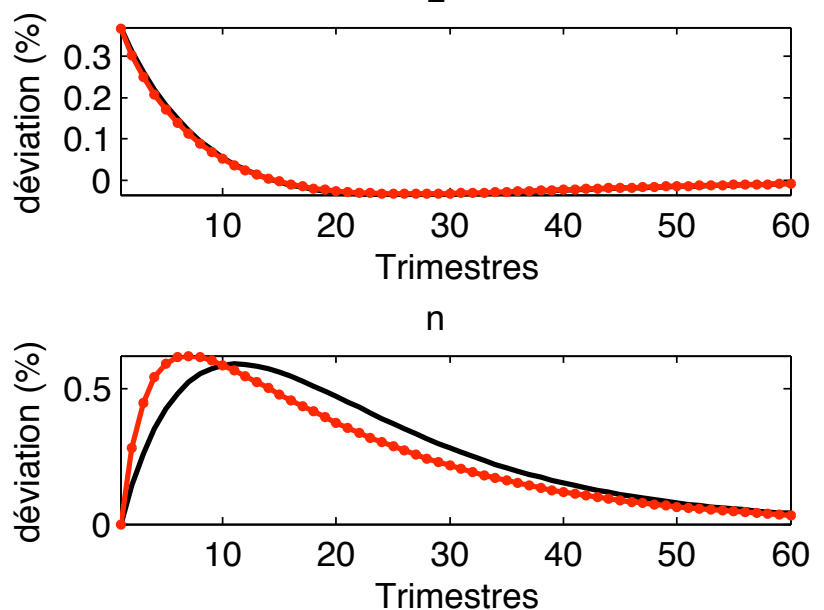

$c^{*}$

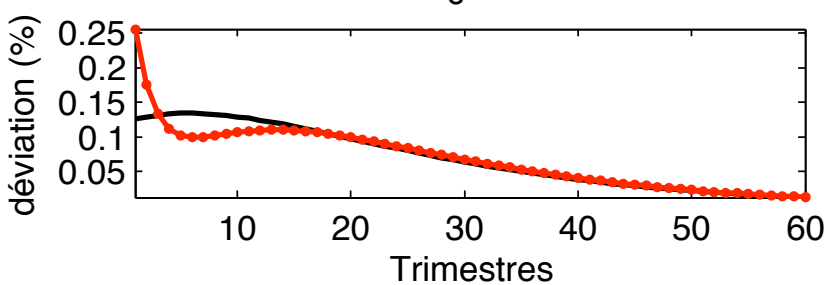

L

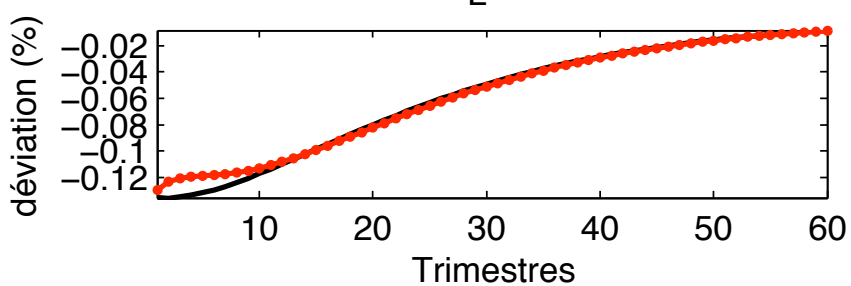

$n^{*}$

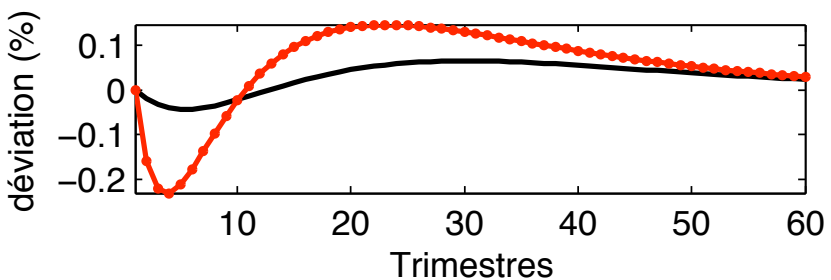


Figure 2 - Réponse à un choc domestique de productivité (2) - taux de change flexible.
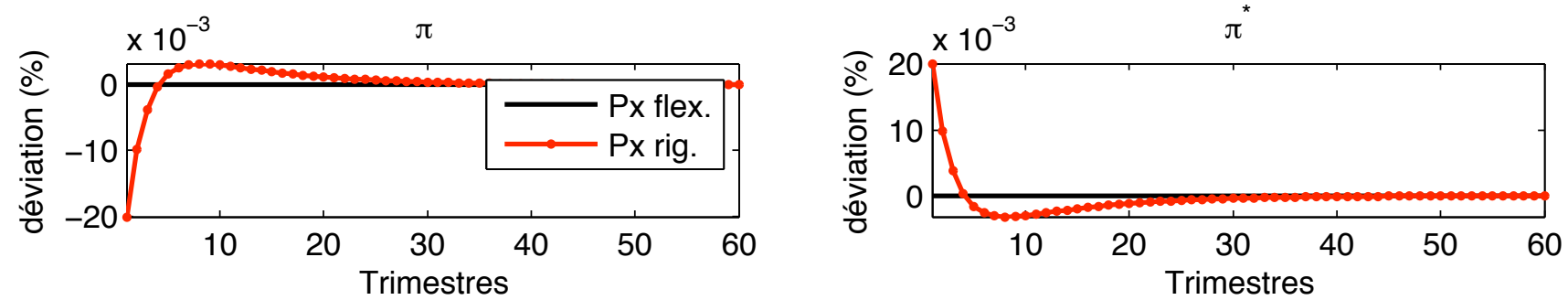

i
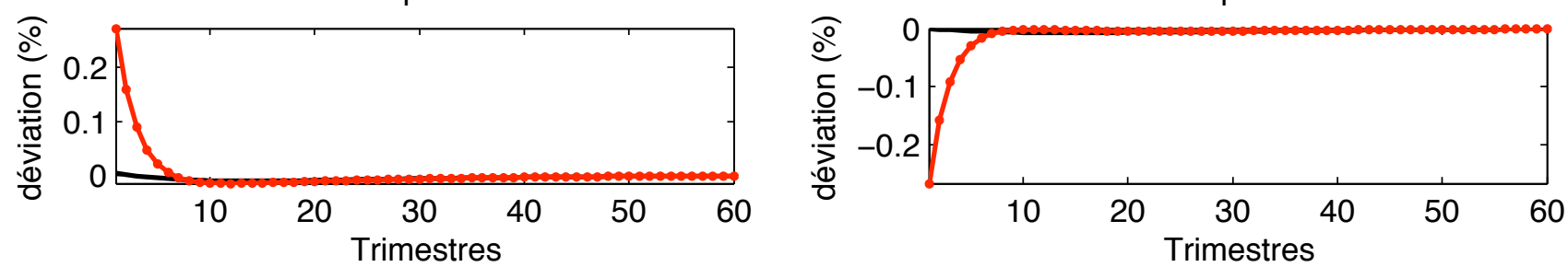

$r$
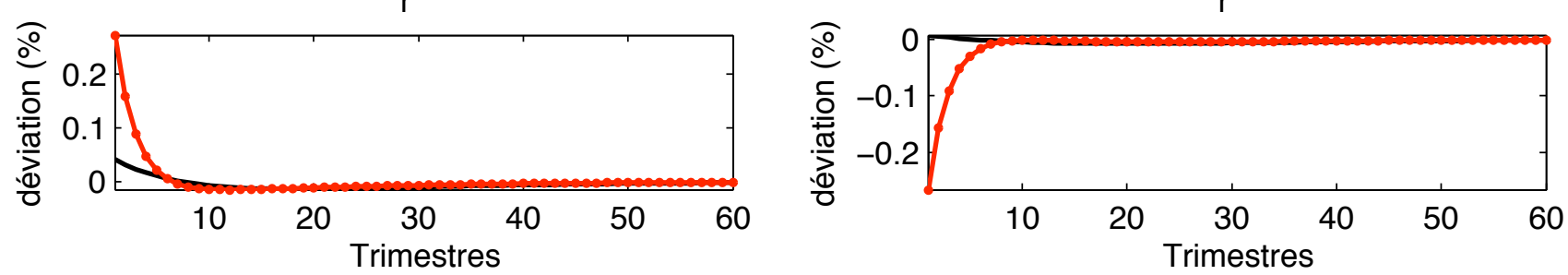

q
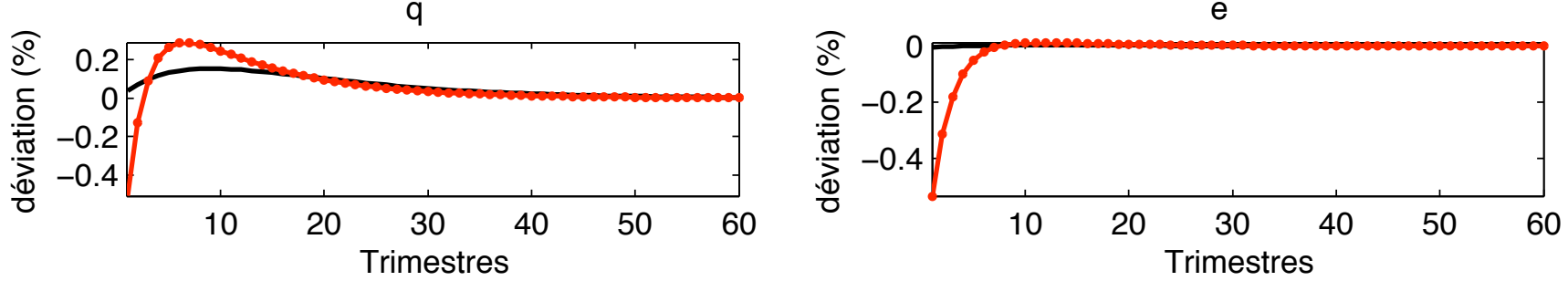
prix de vente. Par conséquent, lorsque les prix sont rigides, les firmes localisées dans l'économie domestique augmentent leurs marges. Toutefois, les coûts d'ajustement payés par les firmes grèvent les profits et l'augmentation des dividendes liée à cette augmentation des marges reste faible. De même, la déflation observée dans l'économie domestique vient réduire la production via le paiement des coûts d'ajustement. La politique monétaire optimale du point de vue de l'économie domestique en change flexible devrait donc consister à stabiliser l'inflation en vue d'amener les heures travaillées et la consommation au plus près de leurs dynamiques de prix flexible. C'est ce que suggère le résultat de Bilbiie et al. [2007] en économie fermée. Toutefois, cette politique n'est pas optimale du point de vue de l'économie mondiale dans son ensemble. En effet, lutter contre l'inflation dans les deux pays simultanément conduirait (à variations de change nominal données) à réduire de manière significative les fluctuations du taux de change réel par le biais de la stabilisation des termes de l'échange. Il existe donc dans ce cadre, même en change flexible, un arbitrage en termes de politique monétaire. La politique de lutte contre l'inflation requiert en effet une réponse positive du taux d'intérêt nominal domestique (comme dans un modèle avec du capital physique, voir par exemple Faia [2008]) lors des 10 premiers trimestres, avant de baisser sensiblement pendant une vingtaine de trimestres. Certes, l'augmentation du taux d'intérêt nominal produit un effet dépressif immédiat sur la consommation mais permet d'accroître la production par le biais des entrées, favorisées par la hausse des rendements associés à la détention d'actions, hausse rendue nécessaire par la condition d'arbitrage entre actifs et permise par l'accroissement sensible des marges des entreprises. En adoptant un raisonnement symétrique, la politique monétaire optimale dans l'économie qui ne subit pas le choc consiste à baisser temporairement le taux d'intérêt nominal en vue de faire baisser le nombre de variétés. Cette dynamique jointe des taux d'intérêt nominaux tend à apprécier la monnaie de l'économie domestique, ce qui ne permettrait pas, si les taux d'inflation étaient entièrement stabilisés, de voir s'apprécier suffisamment le taux de change réel de l'économie domestique. Du point de vue du planificateur bienveillant, il est donc optimal de laisser se développer la déflation des prix à la production dans l'économie domestique et l'inflation dans l'économie étrangère, afin que le taux de change réel fluctue suffisamment. Cet arbitrage de politique monétaire est le même que celui décrit par Benigno [2004] dans le cadre (plus strict) d'une union monétaire. La politique monétaire optimale dans ce cadre vise donc davantage à cibler l'inflation des prix 
à la consommation que celle des prix à la production. La politique monétaire optimale ainsi caractérisée implique donc une sur-réaction des marges intensives et extensives des productions, nécessaire à l'optimisation des trajectoires de consommations et d'heures travaillées dans les deux économies.

Figure 3 - Réponse à un choc domestique de productivité (1) - taux de change fixes.
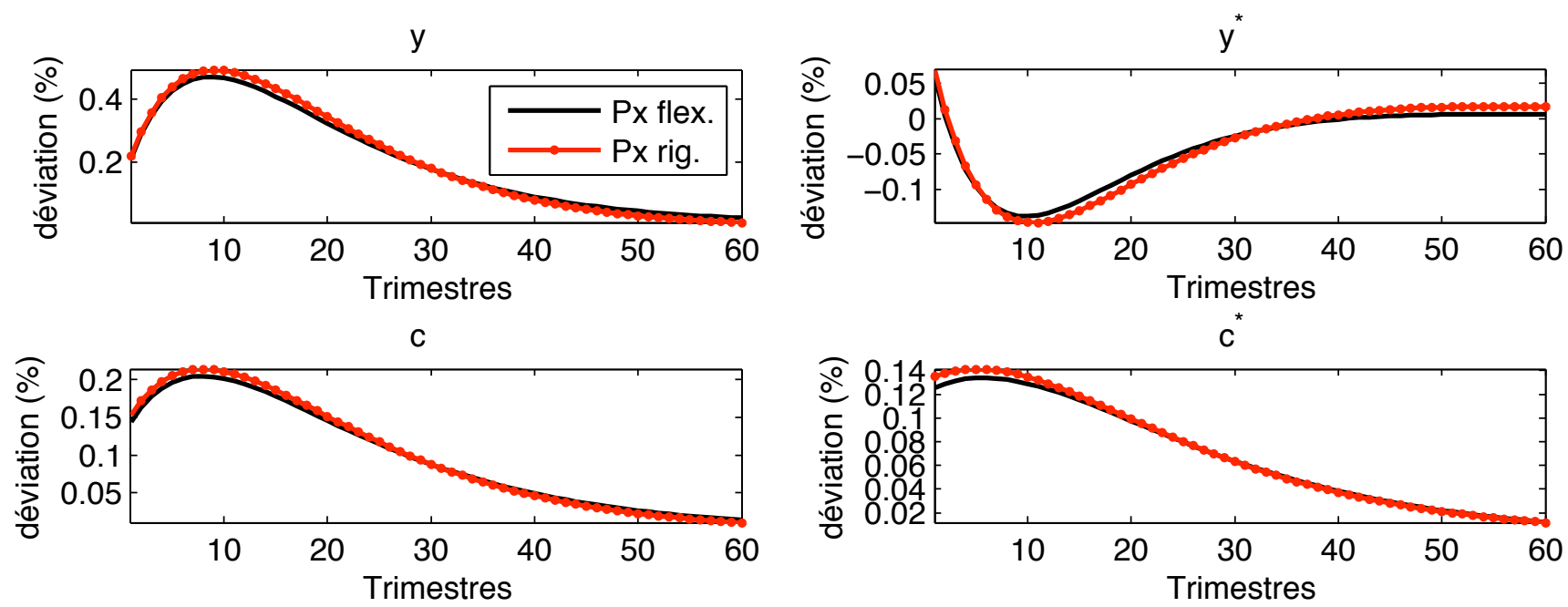

$\mathrm{L}$
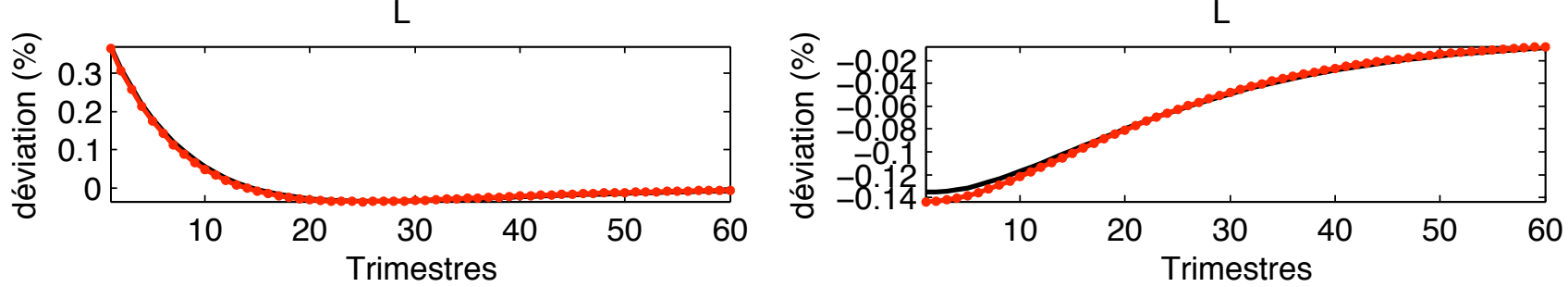

$\mathrm{n}$
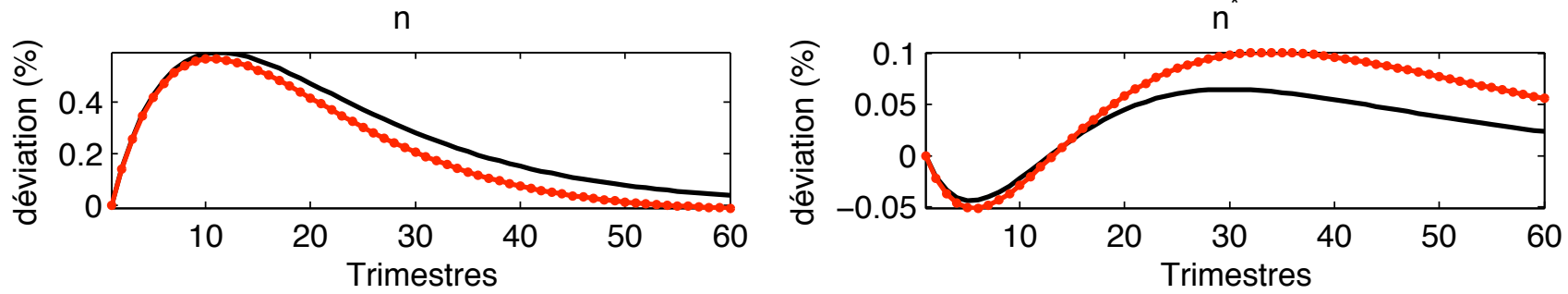

Les graphiques 3 et 4 décrivent la réponse des deux économies en régime de change fixe, i.e. $e_{t}=\bar{e}$, à la suite d'un choc de productivité positif et asymétrique d'1\% se produisant dans l'économie domestique ${ }^{9}$. A des fins de comparaison, les graphiques reportent également la dynamique des économies en régime de change flexible lorsque les prix sont flexibles.

9. L'analyse d'un choc de productivité se produisant dans l'économie étrangère est rigoureusement symétrique. 
FiguRE 4 - Réponse à un choc domestique de productivité (2) - taux de change fixes.
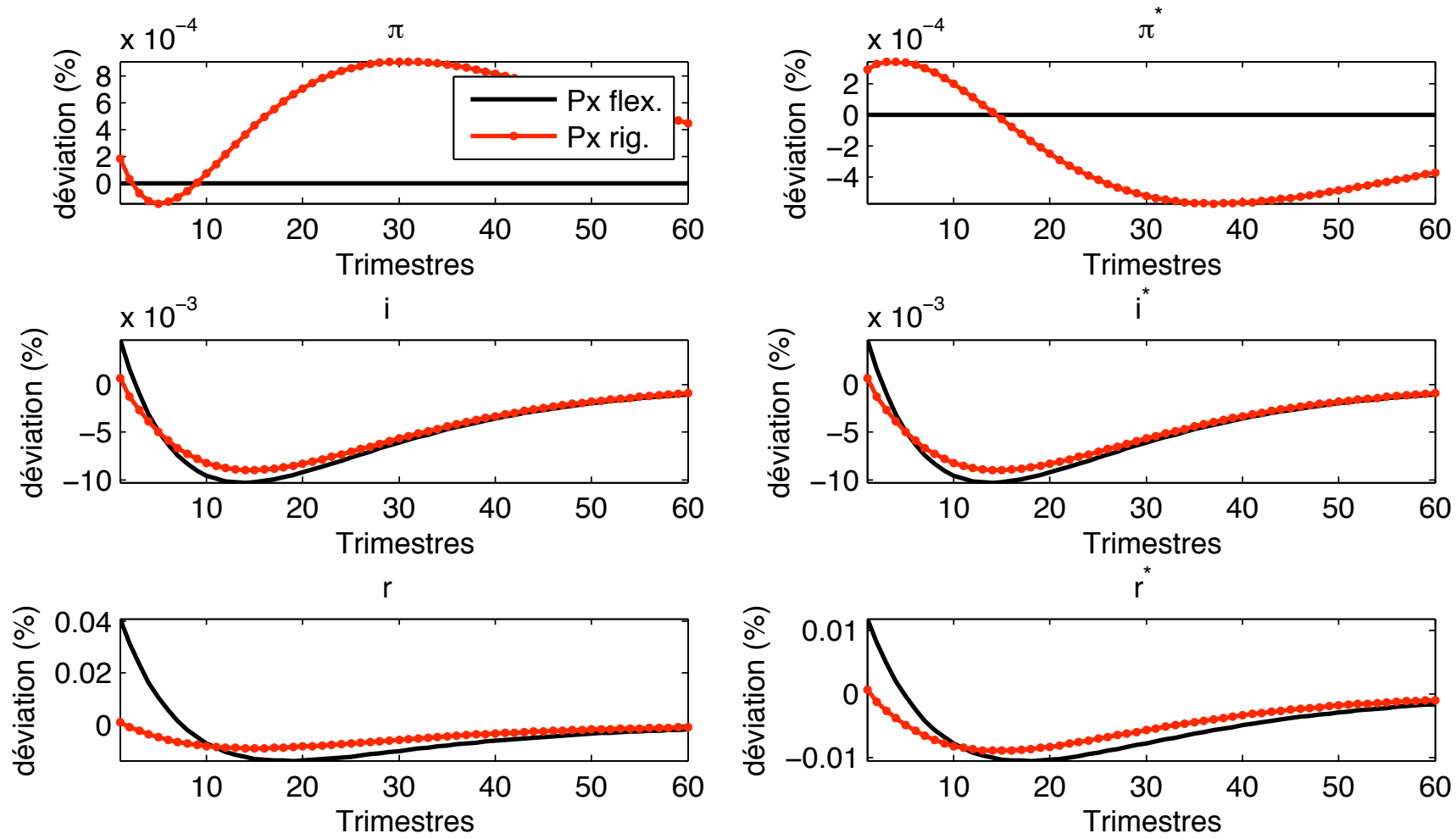

q
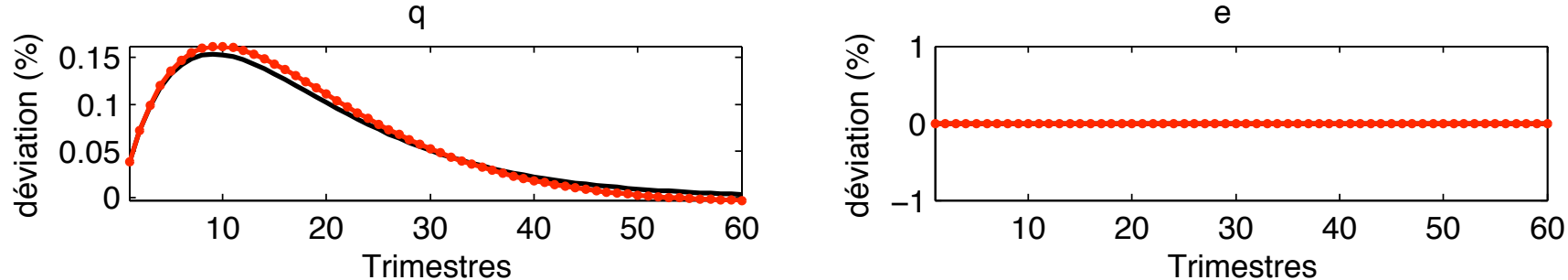
La dynamique des deux économies présente qualitativement de grandes similitudes avec celle obtenue en régime de change flexible en ce qui concerne la dynamique des marges intensives et extensives de la production, la dynamique jointe des consommations et celle des heures travaillées.

Toutefois, on observe que la dynamique du taux d'intérêt nominal, par définition identique dans les deux économies, et celle des taux d'inflation des prix à la production, sont nettement moins réactives que dans le cas précédent. En effet, la dynamique des taux d'intérêt réels est contrainte par celle du taux d'intérêt nominal, qui tente de produire des effets bénéfiques pour les deux économies de manière simultanée, alors même que celles-ci ont besoin de trajectoires de taux d'intérêt nominal différentes. Ainsi l'augmentation du taux d'intérêt réel dans l'économie domestique et la baisse du taux d'intérêt réel dans l'économie étrangère ne permettent pas aux autorités de rapprocher aussi efficacement que dans le cas où le taux de change nominal est flexible les trajectoires de consommations et d'heures travaillées de leurs dynamiques à prix flexibles. La flexibilité du taux de change nominal, en ce qu'elle permet à des économies parfaitement intégrées sur les marchés financiers d'adopter des politiques monétaires indépendantes, est donc susceptible de générer des gains de bien-être conséquents. Cet aspect est d'autant plus important qu'une dimension importante de la dynamique conjoncturelle des économies se joue sur les marchés financiers, via l'impact des taux d'intérêts réel sur la dynamique des taux d'inflation et celle des marges des firmes, qui déterminent la rentabilité de l'activité de création de nouvelles variétés, ayant un effet crucial sur la marge extensive de l'activité.

\section{Analyse de bien-être}

Cette section quantifie les gains en bien-être associés à la flexibilité du taux de change nominal dans un cadre où le nombre de variétés de biens est endogène. Les gains reportés sont, comme nous l'avons déjà évoqué, relatifs à l'indépendance des politiques monétaires menées en cas de chocs asymétriques, et à leurs impacts différenciés sur l'activité à travers les marges intensives et extensives de la production. Comme nous l'avons déjà montré, la rigidité des prix joue un rôle essentiel dans ce cadre puisqu'elle déforme dans le temps l'ajustement des prix aux gains de productivité, affecte ainsi les marges et les profits des entreprises et induit une dynamique inefficace du nombre de variétés disponibles dans les économies. 
En reprenant l'objectif de maximisation du bien-être agrégé, il est possible de montrer qu'une approximation à l'ordre deux des fonctions d'utilité permet de simplifier l'expression de $\Phi_{t}$,

$$
\Phi_{t} \simeq \Phi+\theta_{c}\left(\operatorname{var}\left\{\widehat{c}_{t}\right\}+\operatorname{var}\left\{\widehat{c}_{t}^{*}\right\}\right)-\theta_{\ell}\left(\operatorname{var}\left\{\widehat{\ell}_{t}\right\}+\operatorname{var}\left\{\widehat{\ell}_{t}^{*}\right\}\right)
$$

où $\widehat{c}_{t}=\log \left(c_{t} / c\right)$ et $\widehat{\ell}_{t}=\log \left(\ell_{t} / \ell\right)$, avec $\theta_{c}=\frac{c^{1-\gamma}(1-\gamma)}{2(1-\beta)}$ et $\theta_{\ell}=\frac{c^{1-\gamma}(1+\varphi)(1+\psi)}{2(1-\beta)}$.

Les gains associés à la situation de politique monétaire optimale en régime de change flexible en comparaison de la situation de politique monétaire optimale en régime de change fixe s'évaluent à partir du pourcentage de consommation permanente, noté $\Lambda$, que les ménages sont prêts à abandonner pour vivre dans des économies en change flexible,

$$
\theta_{c}\left(\frac{\Lambda}{100}\right)^{2}=\Phi^{f i x}-\Phi^{f l e x}
$$

Le tableau 1 présente les gains associés à la situation de changes flexibles en comparaison de la situation de changes fixes pour différentes valeurs des paramètres. Il reporte également les écart-types des consommations, des heures travaillées et ceux du taux de change réel pour différentes valeurs des paramètres. Afin d'identifier la contribution de la marge extensive, les mêmes valeurs sont reportées dans la situation où le nombre de variétés est maintenu constant, i.e. $n_{t}=n_{t}^{*}=n$.

Ces résultats montrent que l'adoption d'un régime de change fixe représente un coût en bien-être variant de $0.01 \%$ à $0.2 \%$ de consommation permanente, la calibration de référence faisant apparaître un coût de $0.0296 \%$. Ces résultats sont comparables qualitativement à ceux présentés par Devereux et Engel [2003] et Corsetti [2006] dans un cadre sans marge extensive et quantitativement à ceux de Gali et Monacelli [2005], à la fois en ce qui concerne les volatilités et l'ordre de grandeur des gains de bien-être.

Il est intéressant d'observer que la volatilité du taux de change réel en régime de change flexible lorsque les prix sont rigides est toujours supérieure à celle prévalant lorsque les prix sont flexibles, ce qui renvoie à l'externalité sur le taux de change é voquée précédemment. Au contraire, la volatilité du taux de change réel lorsque les prix sont rigides en régime de change fixe est plus proche de son niveau de prix flexible, au détriment d'une volatilité plus importante des niveaux de consommation et des heures travaillées. Ainsi la variabilité du taux de change réel, 
TABLE 1 - Volatilités et gains de bien-être.

\begin{tabular}{|c|c|c|c|c|c|c|c|}
\hline \multirow{5}{*}{$\begin{array}{l}\text { Référence } \\
\gamma=1.5\end{array}$} & \multicolumn{7}{|c|}{ Prix rigides et marge extensive } \\
\hline & \multicolumn{3}{|c|}{ Changes flexibles } & \multicolumn{3}{|c|}{ Changes fixes } & \multirow[b]{2}{*}{$\Lambda$} \\
\hline & $\sigma\left(c_{t}\right)$ & $\sigma\left(\ell_{t}\right)$ & $\sigma\left(q_{t}\right)$ & $\sigma\left(c_{t}\right)$ & $\sigma\left(\ell_{t}\right)$ & $\sigma\left(q_{t}\right)$ & \\
\hline & 0.81 & 0.60 & 1.02 & 0.81 & 0.62 & 0.66 & 0.0132 \\
\hline & 0.93 & 0.59 & 0.91 & 0.99 & 0.63 & 0.69 & 0.0214 \\
\hline$\gamma=3$ & 0.64 & 0.64 & 1.26 & 0.19 & 0.91 & 0.40 & 0.0261 \\
\hline$\psi^{-1}=2$ & 0.88 & 2.11 & 1.61 & 0.91 & 2.59 & 1.37 & 0.1083 \\
\hline$\psi^{-1}=0.2$ & 0.81 & 0.25 & 0.87 & 0.84 & 0.26 & 0.55 & 0.0113 \\
\hline$\tau=0.25$ & 0.80 & 0.65 & 0.35 & 1.09 & 1.08 & 0.57 & 0.0803 \\
\hline$\tau=0.35$ & 0.79 & 0.64 & 0.54 & 0.95 & 0.84 & 0.61 & 0.0524 \\
\hline
\end{tabular}

Prix rigides sans marge extensive $\left(n_{t}=n_{t}^{*}=n\right)$

\begin{tabular}{lcccccccc} 
& \multicolumn{9}{c}{ Changes flexibles } & & \multicolumn{5}{c}{ Changes fixes } & \\
\cline { 2 - 3 } Référence & $\sigma\left(c_{t}\right)$ & $\sigma\left(\ell_{t}\right)$ & $\sigma\left(q_{t}\right)$ & & $\sigma\left(c_{t}\right)$ & $\sigma\left(\ell_{t}\right)$ & $\sigma\left(q_{t}\right)$ & \\
\cline { 2 - 3 }$\gamma=1.5$ & 0.21 & 0.78 & 0.04 & & 0.20 & 0.86 & 0.04 & 0.0296 \\
$\gamma=3$ & 0.33 & 0.75 & 0.03 & & 0.28 & 0.81 & 0.08 & 0.0256 \\
$\psi^{-1}=2$ & 0.15 & 0.78 & 0.06 & & 0.19 & 0.91 & 0.04 & 0.0361 \\
$\psi^{-1}=0.2$ & 0.46 & 3.00 & 0.28 & & 0.71 & 4.15 & 2.10 & 0.2066 \\
$\tau=0.25$ & 0.11 & 0.32 & 0.02 & & 0.11 & 0.33 & 0.03 & 0.0100 \\
$\tau=0.35$ & 0.22 & 0.75 & 0.00 & & 0.22 & 0.82 & 0.04 & 0.0260 \\
\hline \hline
\end{tabular}

\begin{tabular}{lcccccccc}
\hline \hline & \multicolumn{8}{c}{ Prix flexibles et marge extensive } \\
\cline { 2 - 4 } \cline { 6 - 7 } Référence & \multicolumn{6}{c}{ Changes flexibles } & \multicolumn{5}{c}{ Changes fixes } \\
\cline { 2 - 3 }$\gamma=1.5$ & $\sigma\left(c_{t}\right)$ & $\sigma\left(\ell_{t}\right)$ & $\sigma\left(q_{t}\right)$ & & $\sigma\left(c_{t}\right)$ & $\sigma\left(\ell_{t}\right)$ & $\sigma\left(q_{t}\right)$ & $\Lambda$ \\
$\gamma=3$ & 0.78 & 0.62 & 0.62 & & 0.78 & 0.62 & 0.62 & - \\
$\psi^{-1}=2$ & 0.61 & 0.61 & 0.61 & & 0.91 & 0.61 & 0.61 & - \\
$\psi^{-1}=0.2$ & 0.83 & 2.24 & 1.09 & & 0.83 & 2.24 & 1.09 & - \\
$\tau=0.25$ & 0.76 & 0.26 & 0.51 & & 0.76 & 0.26 & 0.51 & - \\
$\tau=0.35$ & 0.80 & 0.66 & 0.31 & & 0.80 & 0.66 & 0.31 & - \\
\hline
\end{tabular}

$*$ : Tous les chiffres sont en $\%$.

** : Le modèle est symétrique, ce qui implique $\sigma\left(c_{t}^{*}\right)=\sigma\left(c_{t}\right)$ et $\sigma\left(\ell_{t}^{*}\right)=\sigma\left(\ell_{t}^{*}\right)$. 
et donc celle des taux d'inflation des prix à la production, doit être plus forte en vue de mieux contenir la survolatilité des consommations et des heures travaillées, via les effets de l'inflation sur la marge extensive.

De plus, il est notable que l'introduction de la marge extensive peut alternativement augmenter ou réduire les coûts de bien-être liés à l'adoption d'un régime de change fixe. Les gains sont réduits dans le cas de la calibration de référence, pour toutes les variations envisagées de l'élasticité intertemporelle de substitution de la consommation $(\gamma)$ et lorsque l'élasticité frischienne augmente. Les gains augmentent en revanche lorsque l'élasticité frischienne baisse et lorsque les coûts de transport baissent. Ces résultats suggèrent que l'introduction de la marge extensive joue de manière différenciée selon les calibrations sur les variables clés de l'économie. L'introduction de la marge extensive joue sur les heures travaillées via l'équilibre du marché du travail et les heures utilisées afin de créer de nouvelles firmes. Elle affecte également la consommation via les nouvelles variétés introduites et par le biais des échanges commerciaux. Elle réduit fortement la volatilité de la consommation et accroît faiblement la volatilité des heures travaillées.

Lorsque la marge extensive est prise en considération, les consommations et les consommations relatives sont plus volatiles, ce qui entraîne plus de volatilité du taux de change réel par le biais de la relation de partage des risques, quel que soit le régime de change considéré. Par conséquent, l'impact de la fixité du change nominal sur la volatilité des consommations est positif lorsque la marge extensive est prise en compte et négatif lorsqu'elle est ignorée. La volatilité des heures travaillées est cependant toujours renforcée par l'adoption d'un régime de change fixe, et ce plus faiblement lorsque la marge extensive est prise en considération. Par conséquent, lorsque le rapport des élasticités de substitution intertemporelle favorise une forte variabilité des heures travaillées, l'introduction de la marge extensive rend plus faible la hausse de volatilité des heures induite par l'adoption d'un régime de change fixe. Les coûts de bien-être associés sont alors plus faibles. En revanche, lorsque le rapport des élasticités de subsitution intertemporelle favorise une faible variabilité des heures travaillées, cet effet reste trop faible pour compenser l'effet modérateur de l'adoption d'un régime de change fixe sur la volatilité des consommations lorsque la marge extensive est ignorée - alors même que cette volatilité s'accroît lorsque la marge extensive est considérée. Dans ce cas, l'introduction de la marge extensive amplifie les coûts en 
bien-être liés à l'adoption d'un régime de change fixe. Ces mécanismes permettent également de comprendre la manière dont ces coûts sont affectés lorsque les coûts de transport baissent. La baisse des coûts de transport renforce l'intensité de la transmission internationale des chocs asymétriques et tend à accroître les coûts associés à l'adoption d'un régime de change fixe. La hausse de la volatilité des consommations se trouve amplifiée lorsque la marge extensive est considérée, ce qui tend à accroître les coûts d'adoption lorsque la marge extensive est prise en compte par rapport à la situation où elle est ignorée.

Ainsi, la marge extensive rend plus coûteuse l'adoption d'un régime de change fixe lorsque les coûts de transport sont faibles ou lorsque les heures sont faiblement volatiles (de par une faible élasticité frischienne). Elle tend enfin à réduire les coûts de bien-être liés à l'adoption d'un régime de change fixe lorsque les liens commerciaux sont moins intensifs ou lorsque les heures travaillées sont plus volatiles (de par une forte élasticité frischienne).

\section{Conclusion}

Cet article montre que la politique monétaire optimale en économie ouverte avec prise en compte de la marge extensive de l'activité n'est pas la même qu'en économie fermée. La présence d'une externalité sur les termes de l'échange conduit en effet les autorités à laisser les prix à la production fluctuer de manière à assurer une variabilité suffisante pour le taux de change réel. Comme dans le cadre standard, l'adoption d'un régime de change fixe conduit à une survolatilité macroéconomique qui génère des coûts de bien-être. L'introduction de la marge extensive affecte ces coûts. En effet, selon les valeurs des paramètres, les coûts sont réduits ou amplifiés.

\section{Références}

Benigno, P., Optimal Monetary Policy in a Currency Area, Journal of International Economics, 2004, 63 (2), 293-320.

Bergin, P. et C. Lin, Exchange Rate Regimes and the Extensive Margin of Trade, Working Paper 14126, NBER, Cambridge (MA) 2008.

ET G. Consetti, Towards a Theory of Firm Entry and Stabilization Policy, Working Paper 11821, NBER, Cambridge (MA) 2005. 
Bilbile, F.O., F. Ghironi, et M.J. Melitz, Monetary Policy and Business Cycles with Endogenous Entry and Product Variety, in NBER Macroeconomics Annual 2007.

Canzoneri, M., R. Cumby, et B. Diba, The Cost of Nominal Inertia in NNS Models, Journal of Money, Credit and Banking, 2007, 39 (7), 1563-1588.

Consetti, G., Openness and the Case for Flexible Exchange Rates, Research in Economics, 2006, 60 (1), 1-21.

, P. Martin, et P. Pesenti, Productivity, Terms of Trade, and the Home Market Effect, Journal of International Economics, 2007, 73 (1), 99-127.

Devereux, S. Et C. Engel, Monetary Policy in the Open Economy Revisited : Price Setting and Exchange-Rate Flexibility, Review of Economic Studies, 2003, 70 (4), 765-783.

FaiA, E., Ramsey Monetary Policy With Capital Accumulation And Nominal Rigidities, Macroeconomic Dynamics, 2008, 12 (1), 90-99.

Gali, J. et T. Monacelli, Monetary Policy and Exchange Rate Volatility in a Small Open Economy, Review of Economic Studies, 2005, 72 (3), 707-734.

Rotemberg, J., A Monetary Equilibrium Model with Transaction Costs, Journal of Political Economy, 1984, 92 (1), 40-58.

Schmitt-Grohe, S. et M. Uribe, Solving dynamic general equilibrium models using a secondorder approximation to the policy function, Journal of Economic Dynamics and Control, 2004, $28(4), 755-775$.

\section{A Annexe}

\section{A.1 Les conditions d'équilibre du modèle}

Offres de travail

$$
-\frac{u_{\ell, t}}{u_{c, t}}=\frac{\sigma-1}{\sigma} \varpi_{t}, \quad-\frac{u_{\ell^{*}, t}}{u_{c^{*}, t}}=\frac{\sigma-1}{\sigma} \varpi_{t}^{*} .
$$

$\underline{\text { Relations d'Euler sur les bons }}$

$$
\beta E_{t}\left\{\frac{u_{c, t+1}}{u_{c, t}} \frac{1+i_{t+1}}{1+\pi_{c, t+1}}\right\}=1, \quad \beta E_{t}\left\{\frac{u_{c^{*}, t+1}}{u_{c^{*}, t}} \frac{1+i_{t+1}^{*}}{1+\pi_{c, t+1}^{*}}\right\}=1 .
$$


$\underline{\text { Relations d'Euler sur les actions }}$

$v_{t}=\beta(1-\delta) E_{t}\left\{\frac{u_{c, t+1}}{u_{c, t}}\left(d_{t+1}+v_{t+1}\right)\right\}, \quad v_{t}^{*}=\beta(1-\delta) E_{t}\left\{\frac{u_{c^{*}, t+1}}{u_{c^{*}, t}}\left(d_{t+1}^{*}+v_{t+1}^{*}\right)\right\}$.

Dynamiques des variétés

$$
n_{t}=(1-\delta)\left(n_{t-1}+n_{e, t-1}\right), \quad n_{t}^{*}=(1-\delta)\left(n_{t-1}^{*}+n_{e, t-1}^{*}\right)
$$

Tarifications optimales

$$
\rho_{t}=\frac{\sigma}{(\sigma-1)\left(1-\frac{\kappa}{2} \pi_{t}^{2}\right)+\kappa \eta_{t}} \frac{\varpi_{t}}{z_{t}}, \quad \rho_{t}^{*}=\frac{\sigma}{(\sigma-1)\left(1-\frac{\kappa}{2} \pi_{t}^{* 2}\right)+\kappa \eta_{t}^{*}} \frac{\varpi_{t}^{*}}{z_{t}^{*}},
$$

$\underline{\text { Conditions de libre entrée }}$

$$
v_{t}=f_{e} \frac{\varpi_{t}}{z_{t}}, \quad v_{t}^{*}=f_{e} \frac{\varpi_{t}^{*}}{z_{t}^{*}}
$$

Dynamiques des prix relatifs

$$
\frac{1+\pi_{t}}{1+\pi_{c, t}}=\frac{\rho_{t}}{\rho_{t-1}}, \quad \frac{1+\pi_{t}^{*}}{1+\pi_{c, t}^{*}}=\frac{\rho_{t}^{*}}{\rho_{t-1}^{*}}
$$

Effets de variété

$$
n_{t} \rho_{t}^{1-\sigma}+\phi q_{t}^{1-\sigma} n_{t}^{*} \rho_{t}^{* 1-\sigma}=1, \quad n_{t}^{*} \rho_{t}^{* 1-\sigma}+\phi q_{t}^{\sigma-1} n_{t} \rho_{t}^{1-\sigma}=1
$$

$\underline{\text { Equilibres des marchés des biens et services }}$

$$
\frac{y_{t}}{n_{t} \rho_{t}^{1-\sigma}}=c_{t}+\frac{\kappa}{2} \pi_{t}^{2} y_{t}+\phi q_{t}^{\sigma-1}\left(c_{t}^{*}+\frac{\kappa}{2} \pi_{t}^{2} y_{t}^{*}\right), \quad \frac{y_{t}^{*}}{n_{t}^{*} \rho_{t}^{* 1-\sigma}}=c_{t}^{*}+\frac{\kappa}{2} \pi_{t}^{2} y_{t}^{*}+\phi q_{t}^{1-\sigma}\left(c_{t}+\frac{\kappa}{2} \pi_{t}^{2} y_{t}\right) .
$$

Equilibres des marchés du travail

$$
z_{t} \ell_{t}=\frac{y_{t}}{\rho_{t}}+f_{e} n_{e, t}, \quad z_{t}^{*} \ell_{t}^{*}=\frac{y_{t}^{*}}{\rho_{t}^{*}}+f_{e}^{*} n_{e, t}^{*} .
$$


Evolutions des dividendes

$d_{t}=\frac{y_{t}}{n_{t}}\left(1-\frac{\kappa}{2} \pi_{t}^{2}-\frac{(\sigma-1)\left(1-\frac{\kappa}{2} \pi_{t}^{2}\right)+\kappa \eta_{t}}{\sigma}\right), \quad d_{t}^{*}=\frac{y_{t}^{*}}{n_{t}^{*}}\left(1-\frac{\kappa}{2} \pi_{t}^{* 2}-\frac{(\sigma-1)\left(1-\frac{\kappa}{2} \pi_{t}^{* 2}\right)+\kappa \eta_{t}^{*}}{\sigma}\right)$.

$\underline{\text { Dynamique du taux de change réel }}$

$$
\frac{u_{c, t}^{*}}{u_{c, t}}=q_{t}
$$

$\underline{\text { Dynamique du taux de change nominal }}$

$$
\frac{E_{t}\left\{e_{t+1}\right\}}{e_{t}}\left(1+i_{t+1}^{*}\right)=\left(1+i_{t+1}\right) .
$$

\section{A.2 La fonction de bien-être}

La fonction de bien-être s'écrit,

$$
\Phi_{t}=E_{0} \sum_{t=0}^{\infty} \beta^{t}\left\{\int_{0}^{1} u\left(c_{t}(j), \ell_{t}(j)\right) d j+\int_{0}^{1} u\left(c_{t}^{*}(j), \ell_{t}^{*}(j)\right) d j\right\}
$$

Dans la mesure où les agents se comportent de manière symétrique,

$$
\Phi_{t}=E_{0} \sum_{t=0}^{\infty} \beta^{t}\left\{u\left(c_{t}, \ell_{t}\right)+u\left(c_{t}^{*}, \ell_{t}^{*}\right)\right\}
$$

Une approximation à l'ordre deux de cette fonction donne,

$$
\Phi_{t} \simeq E_{0} \sum_{t=0}^{\infty} \beta^{t}\left\{\begin{array}{c}
2 u+c^{1-\gamma}\left(\widehat{c}_{t}+\widehat{c}_{t}^{*}+\frac{(1-\gamma)}{2} \widehat{c}_{t}^{2}+\frac{(1-\gamma)}{2} \widehat{c}_{t}^{* 2}\right) \\
-\ell^{1+\psi}\left(\widehat{\ell}_{t}+\widehat{\ell}_{t}^{*}+\frac{(1+\psi)}{2} \widehat{\ell}_{t}^{2}+\frac{(1+\psi)}{2} \widehat{\ell}_{t}^{* 2}\right)
\end{array}\right\}
$$

où $\widehat{c}_{t}=\log \left(c_{t} / c\right)$ et $\widehat{\ell}_{t}=\log \left(\ell_{t} / \ell\right)$.

Dans la mesure où les situations envisagées n'entraînent jamais de modification de l'état stationnaire, il est possible de simplifier la fonction de la manière suivante,

$$
\Phi_{t} \simeq \Phi+E_{0} \sum_{t=0}^{\infty} \beta^{t}\left\{\begin{array}{c}
c^{1-\gamma}\left(\widehat{c}_{t}+\widehat{c}_{t}^{*}+\frac{(1-\gamma)}{2} \widehat{c}_{t}^{2}+\frac{(1-\gamma)}{2} \widehat{c}_{t}^{* 2}\right) \\
-\ell^{1+\psi}\left(\widehat{\ell}_{t}+\widehat{\ell}_{t}^{*}+\frac{(1+\psi)}{2} \widehat{\ell}_{t}^{2}+\frac{(1+\psi)}{2} \widehat{\ell}_{t}^{* 2}\right)
\end{array}\right\}
$$


En factorisant et en simplifiant, on obtient,

$$
\Phi_{t} \simeq \Phi+c^{1-\gamma} E_{0} \sum_{t=0}^{\infty} \beta^{t}\left\{\begin{array}{c}
\left(\widehat{c}_{t}+\widehat{c}_{t}^{*}+\frac{(1-\gamma)}{2} \widehat{c}_{t}^{2}+\frac{(1-\gamma)}{2} \widehat{c}_{t}^{* 2}\right) \\
-(1+\varphi)\left(\widehat{\ell}_{t}+\widehat{\ell}_{t}^{*}+\frac{(1+\psi)}{2} \widehat{\ell}_{t}^{2}+\frac{(1+\psi)}{2} \widehat{\ell}_{t}^{* 2}\right)
\end{array}\right\}
$$

Enfin, cette expression peut s'approximer par,

$$
\Phi_{t} \simeq \Phi+\frac{c^{1-\gamma}}{1-\beta}\left\{\begin{array}{c}
\left(E\left\{\widehat{c}_{t}\right\}+E\left\{\widehat{c}_{t}^{*}\right\}+\frac{(1-\gamma)}{2} E\left\{\widehat{c}_{t}^{2}\right\}+\frac{(1-\gamma)}{2} E\left\{\widehat{c}_{t}^{* 2}\right\}\right) \\
-(1+\varphi)\left(E\left\{\widehat{\ell}_{t}\right\}+E\left\{\widehat{\ell}_{t}^{*}\right\}+\frac{(1+\psi)}{2} E\left\{\widehat{\ell}_{t}^{2}\right\}+\frac{(1+\psi)}{2} E\left\{\widehat{\ell}_{t}^{* 2}\right\}\right)
\end{array}\right\}
$$

Dans la mesure où l'état stationnaire est efficace, les autorités n'ont pas d'incitation à dévier de l'état stationnaire, ce qui implique, $E\left\{\widehat{c}_{t}\right\}=E\left\{\widehat{c}_{t}^{*}\right\}=E\left\{\widehat{\ell}_{t}\right\}=E\left\{\widehat{\ell}_{t}^{*}\right\}=0$, et permet finalement d'obtenir,

$$
\Phi_{t} \simeq \Phi+\frac{c^{1-\gamma}}{1-\beta}\left\{\frac{(1-\gamma)}{2}\left(\operatorname{var}\left\{\widehat{c}_{t}\right\}+\operatorname{var}\left\{\widehat{c}_{t}^{*}\right\}\right)-\frac{(1+\varphi)(1+\psi)}{2}\left(\operatorname{var}\left\{\widehat{\ell}_{t}\right\}+\operatorname{var}\left\{\widehat{\ell}_{t}^{*}\right\}\right)\right\}
$$




\section{Résumé long}

Cet article étudie la définition de la politique monétaire optimale en économie ouverte lorsque l'évolution de l'activité économique combine la marge intensive - c'est-à-dire la quantité des biens et services produits dans l'économie - et la marge extensive - c'est-à-dire le nombre de variétés produites. Cette dernière composante de l'évolution de l'activité économique liée la création de nouvelles activités (entreprises ou nouveaux produits) apparaît aujourd'hui constituer un élément significatif de la transmission de la politique monétaire, notamment aux Etats-Unis (Bergin et Corsetti, 2005). En effet, dans ce cadre, au-delà de sa fonction traditionnelle dans la stabilisation des prix, la dynamique du taux d'intérêt réel conditionne l'arbitrage intertemporel auquel font face les firmes lorsqu'elles décident d'investir dans la création de nouvelles variétés de biens. Ce faisant elle exerce une influence directe sur le nombre d'entreprises opérant sur le marché. Les travaux effectués en économie fermée (Bilbiie et al., 2007) montrent que, dans ces circonstances, la politique monétaire optimale en économie fermée consiste stabiliser l'inflation des prix la production. L'objet de cet article est d'étendre l'analyse initiale de Bilbiie et al. (2007) au cadre d'une économie ouverte aux échanges réels et financiers internationaux. L'analyse est menée dans le cadre d'un modèle DSGE deux pays de taille identique. Chaque pays est peuplé d'une masse unitaire de consommateurs. Le nombre de firmes opérant dans chaque économie est variable, les entrepreneurs décidant d'entrer sur le marché et de produire en fonction d'une analyse coût avantages. Dans ce cadre, les ménages ont accès trois actifs financiers : les parts d'un fonds mutuel d'investissement national et des bons du trésor émis dans chaque pays. Dans ce cadre théorique, nous nous intéressons la dynamique des économies lorsque la politique monétaire est déterminée de manière optimale. Il s'agit de déterminer la politique monétaire avec engagement préalable qui maximise le bien-être agrégé au niveau international, c'est--dire une politique monétaire coopérative. Cette extension de l'analyse à un modèle DSGE à deux pays permet d'établir deux résultats importants. Tout d'abord, contrairement aux résultats obtenus en économie fermée la simple stabilisation des prix à la production conduit à un ajustement insuffisant du taux de change réel. Ce résultat provient de l'externalité générée par les politiques monétaires sur les termes de l'échange. En effet, une stabilisation parfaite des taux d'inflation des prix à la production conduit à une insuffisante variabilité des termes de l'échange, pourtant nécessaire à l'ajustement externe des économies. Ainsi, l'objectif 
de stabilité des prix à la production n'est plus l'objectif optimal de la politique monétaire en économie ouverte. Le second résultat est relatif aux coûts/bénéfices liés à l'adoption d'un régime de change fixe. Bergin et Lin (2008) montrent que les régimes de change fixe et plus encore les unions monétaires, permettent une réduction de l'incertitude dans le mécanisme de fixation des prix. Ceci conduit alors à une augmentation sensible du niveau des échanges commerciaux par le biais de la marge extensive. Nous ne considérons pas les effets de premier ordre mais nous nous intéressons aux effets des régimes de change sur la volatilit macroconomique. Nous montrons que les régimes de change fixe conduisent à une sur-volatilité macroéconomique, liée la restriction des instruments de politique monétaire, et ce même lorsque la politique monétaire commune est fixée de manire coopérative. Ainsi, les coûts d'adoption d'un régime de change fixe représentent un coût en termes de bien-être représentant entre $0.01 \%$ et $0.1 \%$ de consommation permanente selon les valeurs des paramètres. Ces résultats relatifs la supériorité des régimes de change flexible rejoignent qualitativement et quantitativement ceux déjà publiés dans la littérature. La prise en considération de la marge extensive est donc susceptible de réduire ou d'augmenter les coûts d'adoption d'un système de change fixe par rapport l'analyse standard. Ces résultats sont obtenus en supposant que les termes de l'échange jouent un rôle central dans l'ajustement des flux commerciaux internationaux. Une extension intéressante de l'analyse proposée consisterait à adopter une structure plus réaliste des marchés financiers, en les supposant incomplets. Cette hypothèse aurait pour conséquence de modifier les conditions d'ajustement externe des économies et permettrait probablement de nuancer les résultats obtenus. 